\title{
CPT and lepton number violation in neutrino sector: Modified mass matrix and oscillation due to gravity
}

\author{
Monika Sinha* \\ Theory Division, Saha Institute of Nuclear Physics \\ 1/AF Bidhannagar, Kolkata 700 064, India. \\ Banibrata Mukhopadhyay \\ Department of Physics, Indian Institute of Science \\ Bangalore 560012, India.
}

\begin{abstract}
We study the consequences of CPT and lepton number violation in neutrino sector. For CPT violation we take gravity with which neutrino and antineutrino couple differently. Gravity mixes neutrino and antineutrino in an unequal ratio to give two mass eigenstates. Lepton number violation interaction together with CPT violation gives rise to neutrino-antineutrino oscillation. Subsequently, we study the neutrino flavor mixing and oscillation under the influence of gravity. It is found that gravity changes flavor oscillation significantly which influences the relative abundance of different flavors in present universe. We show that the neutrinoless double beta decay rate is modified due to presence of gravity - the origin of CPT violation, as the mass of the flavor state is modified.

PACS numbers: 14.60.Pq; 14.60.St; 23.40.-s; 96.12.Fe
\end{abstract}

\section{INTRODUCTION}

The oscillations between different kinds of neutrino and antineutrino flavor have been observed form solar, atmospheric and LSND data. The three pieces of observation indicate three values of mass-squared difference of three different orders. With three families of neutrino, one can obtain only two independent mass-squared differences. Therefore, observations require the introduction of fourth neutrino which must be sterile in the standard model. But many difficulties arise with the introduction of fourth neutrino as discussed in literature (e.g. see [1]).

As an alternate proposal to accommodate the results, many authors have proposed CPT violation in neutrino sector [2, 3, 4]. One can either introduce a new particle (sterile neutrino) or allow CPT violation to take care of all experimental results with present data. However, very recently, MiniBooNE results have been declared which shows that LSND experimental results can not be explained simply by neutrino oscillation. Hence, it raises many other questions to be answered [5].

Previously, many authors have explored the consequences of CPT violation in neutrino sector (e.g. see [6, 7]). The nature of mass of neutrino has been studied [7] if CPT is violated. It has been shown that if CPT is violated then neutrinos no longer remain Majorana particles even if they have a Majorana mass i.e. they violate lepton number. But the actual physical condition which can lead to CPT violation was not discussed satisfactorily. Proper situations in which CPT can be violated were discussed later by many authors [8, 9, 10, 11, 12, 13, 14]. They have pointed out that CPT violation in neutrino sector can occur due to the spin-gravity coupling.

In the present paper, we plan to obtain the mass matrix for neutrino sector with Majorana mass in the presence of gravity. It has already been seen that spin gravity coupling has an extra contribution to the effective mass of neutrino and antineutrino. The Majorana type mass of neutrino alone can cause lepton number violation i.e. the mixing of neutrino and antineutrino states. Without an interaction with gravity, the neutrino-antineutrino mixing angle is $\pi / 4$. However, in presence of background gravitational field, mixing angle changes and depends on the strength of CPT violation. In a similar fashion, flavor oscillation is also altered by gravitational effect. As a consequence of mixing, the neutrino-antineutrino oscillation can take place only in presence of gravity. We also show that gravity affects the neutrinoless double beta decay rate even if two neutrino flavor mixing is taken.

We organize the paper in the following manner. In the next section, we review the CPT violating nature of the spingravity interaction for neutrino. In the section III we obtain the mass matrix for neutrino traveling in a background gravitational field. From that we find the neutrino-antineutrino mixing and oscillation. Subsequently, we investigate effect of gravity on flavor oscillation and its application to that in early universe and around black holes in the section

*Electronic address: monika.sinha@saha.ac.in

${ }^{\dagger}$ Electronic address: bm@physics.iisc.ernet.in 
IV. Applying this mixing, we recalculate the neutrinoless double beta decay rate in the next section. Finally, we summarize what we obtain and discuss their implications in the section VI.

\section{COUPLING TO CURVATURE}

The CPT violation mechanism due to the spin-gravity coupling of fermions has been discussed earlier [8, 9, 10, 13. How differently gravity interacts with neutrino than antineutrino has been shown by a detailed calculation [10, 13, 14]. For completeness, here we revisit the idea very briefly.

The general invariant coupling of spin-1/2 particles to gravity is described by the Lagrangian [15, 16, 17, 18, 19, $20,21,22,23]$

$$
\mathcal{L}=\sqrt{-g}\left(\frac{i}{2} \bar{\Psi} \gamma^{a} \stackrel{\leftrightarrow}{D}_{a} \Psi-\bar{\Psi} m \Psi\right)
$$

where the covariant derivative is

$$
D_{a}=\left(\partial_{a}-\frac{i}{4} \omega_{b c a} \sigma^{b c}\right)
$$

and the spin-connections are

$$
\omega_{b c a}=e_{b \lambda}\left(\partial_{a} e_{c}^{\lambda}+\Gamma_{\gamma \mu}^{\lambda} e_{c}^{\gamma} e_{a}^{\mu}\right) .
$$

Here

$$
\sigma^{b c}=\frac{i}{2}\left[\gamma^{b}, \gamma^{c}\right]
$$

All the above equations have been written in a local inertial frame which is flat along the entire geodesic. The Roman alphabets denote the flat space indices, while the Greek alphabets denote the curved space indices. Here $e$-s are the vierbeins connecting curved and locally flat spaces and obey the relations $e_{a}^{\mu} e^{\nu a}=g^{\mu \nu}, e^{a \mu} e_{\mu}^{b}=\eta^{a b}$, where $\eta^{a b}$ represents the inertial frame Minkowski metric and $g^{\mu \nu}$ the curved spacetime metric.

Thus the Lagrangian can be rewritten as

$$
\mathcal{L}=\operatorname{det}(e) \bar{\Psi}\left(\frac{i}{2} \gamma^{a} \stackrel{\leftrightarrow}{\partial}_{a}-m+\gamma^{a} \gamma^{5} B_{a}\right) \Psi
$$

with

$$
B^{d}=\epsilon^{a b c d} \omega_{b c a}
$$

In a local inertial frame, the effect of gravity on fermion is only an axial vector interaction term involving the gravitational field $B_{a}$ given by Eq. (6). The Lagrangian given by Eq. (15) has two parts- the free part and the interaction part. The interaction part is a coupling to the field $B_{a}$ which is constant in a local inertial frame because that arises from the background gravitational field.

If $B_{a}$ is constant in a local inertial frame, then the interaction term violates CPT as well as particle Lorentz symmetry, although it is invariant under observer Lorentz transformation (see e.g. [24]). It has been shown that only a special form of the background gravitational field can give rise to such CPT violating interaction term. For example, around the rotating black holes [9, 10], or in anisotropic early universe [13], a suitable background field exists which causes CPT violation in interaction with spin- $1 / 2$ fermions.

The interaction term in the Lagrangian given by Eq. (5) involving $B_{a}$ contains $\gamma^{5}$. If we consider the standard model neutrino with the Majorana mass term, then right-handed neutrino and left handed antineutrino are absent. This leads the gravitational interaction to $-\bar{\Psi}_{L} \gamma^{a} \Psi_{L}$ and $\bar{\Psi}_{L}^{c} \gamma^{a} \Psi_{L}^{c}$ for neutrino and antineutrino respectively. Here $c$ superscripted $\Psi$ represents the charge conjugated spinor or the spinor for antiparticle and the subscript $L$ denotes the left handed component. Consequently, the dispersion relations for neutrino and antineutrino become different respectively given as

$$
\begin{aligned}
& E_{\nu}=\sqrt{(\vec{p}-\vec{B})^{2}+m^{2}}+B_{0}, \\
& E_{\nu^{c}}=\sqrt{(\vec{p}+\vec{B})^{2}+m^{2}}-B_{0}
\end{aligned}
$$

with momentum $\vec{p}$. 


\section{MASS MATRIX}

If we consider Majorana neutrino, then in Weyl representation neutrino spinor can be written as

$$
\Psi=\left(\begin{array}{c}
\psi_{L}^{c} \\
\psi_{L}
\end{array}\right)
$$

where $\psi_{L}^{c}$ and $\psi_{L}$ are two component spinors for antineutrino and neutrino respectively which are lepton number eigenstates with eigenvalues -1 and +1 respectively. Here we assume that neutrino is a left handed particle only. Hence, hereafter we shall omit the subscript $L$.

In terms of two component spinors, the Majorana mass term can be written as

$$
\begin{array}{r}
\bar{\Psi}^{c} M \Psi=\left(\begin{array}{ll}
\psi^{c \dagger} & \psi^{\dagger}
\end{array}\right)\left(\begin{array}{cc}
0 & -m \\
-m & 0
\end{array}\right)\left(\begin{array}{c}
\psi^{c} \\
\psi
\end{array}\right) \\
=-\psi^{c \dagger} m \psi-\psi^{\dagger} m \psi^{c} .
\end{array}
$$

Now in gravitational field the Lagrangian density can be written as

$$
\begin{array}{r}
(-g)^{-1 / 2} \mathcal{L}=\left(\begin{array}{ll}
\psi^{c \dagger} \psi^{\dagger}
\end{array}\right) \frac{i}{2} \gamma^{0} \gamma^{\mu} \stackrel{\leftrightarrow}{\mathcal{D}}_{\mu}\left(\begin{array}{c}
\psi^{c} \\
\psi
\end{array}\right)+\left(\begin{array}{ll}
\psi^{c \dagger} & \psi^{\dagger}
\end{array}\right) \gamma^{5} B_{0}\left(\begin{array}{c}
\psi^{c} \\
\psi
\end{array}\right) \\
-\left(\begin{array}{ll}
\psi^{c \dagger} & \psi^{\dagger}
\end{array}\right) \gamma^{0} m\left(\begin{array}{c}
\psi^{c} \\
\psi
\end{array}\right) \\
=\left(\begin{array}{lll}
\psi^{c \dagger} & \psi^{\dagger}
\end{array}\right) \frac{i}{2} \gamma^{0} \gamma^{\mu} \stackrel{\leftrightarrow}{\mathcal{D}}_{\mu}\left(\begin{array}{c}
\psi^{c} \\
\psi
\end{array}\right)+\left(\begin{array}{ll}
\psi^{c \dagger} & \psi^{\dagger}
\end{array}\right)\left(\begin{array}{cc}
B_{0} & 0 \\
0 & -B_{0}
\end{array}\right)\left(\begin{array}{c}
\psi^{c} \\
\psi
\end{array}\right) \\
-\left(\begin{array}{ll}
\psi^{c \dagger} & \psi^{\dagger}
\end{array}\right)\left(\begin{array}{cc}
0 & -m \\
-m & 0
\end{array}\right)\left(\begin{array}{c}
\psi^{c} \\
\psi
\end{array}\right)
\end{array}
$$

where

$$
\mathcal{D}_{\mu} \equiv\left(\partial_{0}, \partial_{i}+\gamma^{5} B_{i}\right)
$$

Therefore, in Lagrangian density we obtain terms containing $B_{0} \psi^{c \dagger} \psi^{c}$ and $B_{0} \psi^{\dagger} \psi$. These two terms do not violate lepton number. Hence these terms can be looked as lepton number non-violating mass of Majorana neutrino. Therefore, writing the mass terms together we obtain the Lagrangian density

$$
(-g)^{-1 / 2} \mathcal{L}=\left(\begin{array}{ll}
\psi^{c \dagger} & \psi^{\dagger}
\end{array}\right) \frac{i}{2} \gamma^{0} \gamma^{\mu} \stackrel{\leftrightarrow}{\mathcal{D}}_{\mu}\left(\begin{array}{c}
\psi^{c} \\
\psi
\end{array}\right)-\left(\begin{array}{ll}
\psi^{c \dagger} & \psi^{\dagger}
\end{array}\right)\left(\begin{array}{cc}
-B_{0} & -m \\
-m & B_{0}
\end{array}\right)\left(\begin{array}{c}
\psi^{c} \\
\psi
\end{array}\right)
$$

Hence, in background gravitational field the Euler-Lagrange equation for neutrino and antineutrino is

$$
i \gamma^{0} \gamma^{\mu} \mathcal{D}_{\mu}\left(\begin{array}{c}
\psi^{c} \\
\psi
\end{array}\right)-\left(\begin{array}{cc}
-B_{0} & -m \\
-m & B_{0}
\end{array}\right)\left(\begin{array}{c}
\psi^{c} \\
\psi
\end{array}\right)=0
$$

Therefore, the mass matrix of the neutrino-antineutrino sector is given by

$$
\mathcal{M}=\left(\begin{array}{cc}
-B_{0} & -m \\
-m & B_{0}
\end{array}\right)
$$

Hence we see that if one assumes neutrino to have solely the Majorana type masses, then it acquires lepton number non-violating type masses, equal but opposite in sign, while propagating in a gravitational field. Moreover, we see that in this case $\psi$ no longer remains a mass eigenstate.

\section{A. Neutrino-antineutrino mixing and oscillation}

The mass matrix $\mathcal{M}$, given by Eq. (14), is Hermitian and can be diagonalized by unitary transformation. Then the mass eigenstates will be some admixture of $\psi$ and $\psi^{c}$. Hence, neutrino and antineutrino states couple together. 
We find these two mass eigenstates $\nu_{1}$ and $\nu_{2}$ as

$$
\begin{array}{r}
\left|\nu_{1}\right\rangle=\frac{1}{N}\left\{\left(B_{0}+\sqrt{B_{0}^{2}+m^{2}}\right)\left|\psi^{c}\right\rangle+m|\psi\rangle\right\} \\
\left|\nu_{2}\right\rangle=\frac{1}{N}\left\{-m\left|\psi^{c}\right\rangle+\left(B_{0}+\sqrt{B_{0}^{2}+m^{2}}\right)|\psi\rangle\right\} .
\end{array}
$$

with eigenvalues

$$
m_{1,2}=\mp \sqrt{B_{0}^{2}+m^{2}}
$$

Here

$$
N=\sqrt{2 B_{0}^{2}+2 m^{2}+2 B_{0} \sqrt{B_{0}^{2}+m^{2}}}
$$

is the normalization factor. In a more convenient way, one can write

$$
\begin{array}{r}
\left|\nu_{1}\right\rangle=\cos \theta\left|\psi^{c}\right\rangle+\sin \theta|\psi\rangle \\
\left|\nu_{2}\right\rangle=-\sin \theta\left|\psi^{c}\right\rangle+\cos \theta|\psi\rangle
\end{array}
$$

with

$$
\tan \theta=\frac{m}{B_{0}+\sqrt{B_{0}^{2}+m^{2}}} .
$$

Then one can write $\left|\psi^{c}\right\rangle$ and $|\psi\rangle$ as the superposition states of $\left|\nu_{1}\right\rangle$ and $\left|\nu_{2}\right\rangle$ in the following manner

$$
\begin{gathered}
\left|\psi^{c}\right\rangle=\cos \theta\left|\nu_{1}\right\rangle-\sin \theta\left|\nu_{2}\right\rangle \\
|\psi\rangle=\sin \theta\left|\nu_{1}\right\rangle+\cos \theta\left|\nu_{2}\right\rangle .
\end{gathered}
$$

Basically, $\nu_{1}=-i \sigma_{2} \nu_{2}^{*}$ as $\psi^{c}=-i \sigma_{2} \psi$. Therefore, $\nu_{1}$ and $\nu_{2}$ together actually describe a single Majorana particle as $\psi$ and $\psi^{c}$ do. Then, if we construct a new four component neutrino spinor as

$$
\nu \equiv\left(\begin{array}{l}
\nu_{1} \\
\nu_{2}
\end{array}\right)
$$

it retains Majorana nature, i.e. $\nu^{c}=\nu$. This is obvious as the new spinor $\nu$ is only a transformed spinor from the previous one $\Psi$ and the old and new spinors are related by a unitary transformation

$$
\nu=\left(\begin{array}{cc}
\cos \theta & \sin \theta \\
-\sin \theta & \cos \theta
\end{array}\right) \Psi
$$

Although, $\nu_{1}$ and $\nu_{2}$ are not lepton number eigenstates, as evident from Eq. (15), the mass terms in the corresponding Lagrangian of $\nu_{1}$ and $\nu_{2}$ are lepton number conserving. In absence of gravitational field, neutrino and antineutrino mix in the same angle.

Hence, if there is any lepton number violating interaction, then we obtain two neutrino mass eigenstates $\nu_{1}$ and $\nu_{2}$ which are superpositions of neutrino and antineutrino states given by Eq. (18). As energies of neutrino and antineutrino in gravitational field are different, there will be an oscillation between $\nu_{1}$ and $\nu_{2}$. At any time $t$, the oscillation probability is given by

$$
\mathcal{P}(t)=\sin ^{2} 2 \theta \sin ^{2} \delta(t)
$$

where

$$
\delta(t)=\frac{\left|E_{\nu}-E_{\nu^{c}}\right| t}{2},
$$

when $E_{\nu}$ and $E_{\nu^{c}}$ are given by Eq. (7). Hence, for ultra relativistic neutrinos by putting the value of $\theta$, we obtain

$$
\mathcal{P}(t)=\frac{m^{2}}{B_{0}^{2}+m^{2}} \sin ^{2}\left\{\left(B_{0}-|\vec{B}|\right) t\right\}
$$


for neutrino and antineutrino of same rest mass $m$. Thus the oscillation length is

$$
\lambda=\frac{\pi}{B_{0}-|\vec{B}|} .
$$

This depends only on the strength of the gravitational field. If we consider neutrinos to be coming out off the inner accretion disk around a spinning black hole of mass $M=10 M_{\odot}$, then $B_{0}-|\vec{B}|=\tilde{B}=10^{-19} \mathrm{GeV}$ [14], which leads to $\lambda \sim 10 \mathrm{~km}$. If the disk is around a supermassive black hole of $M=10^{8} M_{\odot}$ in an AGN, then $\lambda$ may increase to $10^{10} \mathrm{~km}$, depending upon the size of inner edge where from neutrinos come out and angular momentum of the black hole. Therefore, an oscillation may complete from a few factor to hundred Schwarzschild radii in the disk producing copious antineutrino over neutrino and may cause overabundance of neutron and positron. However, neutrinos around a primordial black hole of mass $M_{p}$ [38] could lead to an oscillation length as small as $\lambda \sim 10^{-16} \mathrm{~km} \leq 100 M_{p}$ for $r \leq 100 M_{p}$.

Therefore, from Eqs. (18) and (19) we see that for nonzero value of Majorana mass $(m \neq 0)$, neutrino and antineutrino combine to give two new states $\nu_{1}$ and $\nu_{2}$, mass eigenstates with different mass eigenvalues. Therefore, in these two states neutrino and antineutrino are mixed. In the absence of gravity, the mixing angle is $\pi / 4$ which is evident from the Eq. (19). On the other hand, if $m=0$, i.e. there is no lepton number violating interaction, then neutrino and antineutrino do not couple at all [see Eq. (13)]. This means two component neutrino and antineutrino described in Eq. (8) themselves are mass eigenstates. In this case, presence of gravity which is CPT violating, splits these two eigenstates with two different mass eigenvalues.

We see that although initially gravitational interaction and the Majorana mass term explicitly have different effects, one to violate $\mathrm{CPT}$ and another to violate lepton number, both of them contribute in the same manner to split the mass eigenstates (see Eq. (16)). Moreover, in gravitational field, since neutrino and antineutrino acquire different effective masses, gravitational field coupled to neutrino spin may have some lepton number violating nature implicitly. This has been illustrated in literature [10, 13]. We also see from Eq. (25) that presence of gravity leads to oscillation. Without gravitational field, the lepton number violating interaction alone can not cause this oscillation.

\section{B. Oscillation with lepton number conserving mass}

For Majorana neutrino when the sterile components are neglected, the Dirac mass is of no relevance. This is exactly the case we are considering in our present paper. We have also seen that the gravity can induce an effective mass for Majorana neutrino which does not violate lepton number. If we consider the diagonal term of the mass matrix of Eq. (9) to be non-zero, then we obtain an extra mass term which conserves the lepton number. With this mass term included, the effective mass matrix will take the form

$$
\mathcal{M}_{n}=\left(\begin{array}{cc}
m_{n}-B_{0} & -m \\
-m & m_{n}+B_{0}
\end{array}\right)
$$

where $m_{n}$ is the so called lepton number non-violating mass.

With this mass matrix, the mixing angle of neutrino and antineutrino does not alter. However, the mass eigenvalues of the mass eigenstates become

$$
m_{n(1,2)}=m_{n} \mp \sqrt{B_{0}^{2}+m^{2}} .
$$

In this case, we can consider oscillation between neutrino and antineutrino as they are combination of $\nu_{1}$ and $\nu_{2}$ which evolve differently with time. For this oscillation, the oscillation probability at any time $t$ is given by

$$
\mathcal{P}_{n}(t)=\sin ^{2} 2 \theta \sin ^{2} \epsilon(t)
$$

where

$$
\epsilon(t)=\frac{\left|E_{1}-E_{2}\right| t}{2},
$$

when $E_{1}$ and $E_{2}$ are energies of two neutrino mass eigenstates with momentum $\vec{p}$. In the ultra-relativistic limit $(|\vec{p}|>>m)$

$$
E_{(1,2)}=|\vec{p}|+\frac{m_{n(1,2)}^{2}}{2|\vec{p}|} .
$$


Again assuming $|\vec{p}| \sim E$

$$
E_{2}-E_{1}=\frac{m_{n 2}^{2}-m_{n 1}^{2}}{2 E}=\frac{2 m_{n} \sqrt{B_{0}^{2}+m^{2}}}{E}
$$

Hence the the oscillation probability at any time $t$ is given by

$$
\mathcal{P}_{n}(t)=\frac{m^{2}}{B_{0}^{2}+m^{2}} \sin ^{2}\left(\frac{m_{n} \sqrt{B_{0}^{2}+m^{2}}}{E} t\right)
$$

and the oscillation length is given by

$$
\lambda_{n}=\frac{\pi E}{m_{n} \sqrt{B_{0}^{2}+m^{2}}} .
$$

Hence we see that this neutrino-antineutrino oscillation probability does not depend on the spatial part of the gravitational field (gravitational vector potential) $\vec{B}$ but depends on the temporal part (gravitational scalar potential) $B_{0}$ only. However, there is nothing new in it. It was already shown that neutrino asymmetry and then leptogenesis in early universe arises due to non-zero $B_{0}[13]$ independent of $\vec{B}$. The gravitational scalar potential $B_{0}$ is non-zero if the background spacetime has at least one nonzero off-diagonal spatial component when the set of coordinate variables is $\{t, x, y, z\}$. Existence of such a component in a spacetime may be due to anisotropy which is the case for the Bianchi model. Moreover, off-diagonal spatial components also govern in presence of primordial quantum fluctuations in the Robertson-Walker spacetime. This is basically the tensor perturbation to early universe. Therefore, it is confirmed that the gravity induced leptogenesis is possible only if the gravitational scalar potential is non-zero.

\section{EFFECT OF GRAVITY ON FLAVOR MIXING AND OSCILLATION}

\section{A. Flavor mixing}

In previous section we have seen that interaction of neutrino and antineutrino with gravity gives rise to neutrino mass eigenstates which are superposition of neutrino and antineutrino states given by

$$
\nu_{e, \mu}=\mathcal{U}_{e, \mu}^{\dagger} \Psi_{e, \mu}
$$

where

$$
\mathcal{U}_{e, \mu}=\left(\begin{array}{cc}
\cos \theta_{e, \mu} & -\sin \theta_{e, \mu} \\
\sin \theta_{e, \mu} & \cos \theta_{e, \mu}
\end{array}\right)
$$

It is clear from our above discussions that $\nu_{e, \mu}$ here are four component spinors. In other way, it can be stated that in gravitational field neutrino state $\Psi$ of mass $m$ is modified to the state $\nu$ of mass $\sqrt{B_{0}^{2}+m^{2}}$ as described in section III. Therefore, recalling Eq. (21) we construct flavor eigenstates $\nu_{e}$ and $\nu_{\mu}$ under gravity for electron and muon neutrino respectively whose components are $\nu_{e 1}, \nu_{e 2}, \nu_{\mu 1}$ and $\nu_{\mu 2}$ with masses $m_{e 1}, m_{e 2}, m_{\mu 1}$ and $m_{\mu 2}$ respectively. In terms of gravitational coupling these mass eigenvalues are expressed as

$$
\begin{array}{r}
m_{(e, \mu) 1}=-\sqrt{B_{0}^{2}+m_{e, \mu}^{2}}, \\
m_{(e, \mu) 2}=\sqrt{B_{0}^{2}+m_{e, \mu}^{2}},
\end{array}
$$

where $m_{e}, m_{\mu}$ are the Majorana masses for electron and muon neutrino respectively, analogous to $m$ of section III. The corresponding mixing parameters are given by

$$
\tan \theta_{e, \mu}=\frac{m_{e, \mu}}{B_{0}+\sqrt{B_{0}^{2}+m_{e, \mu}^{2}}} .
$$

Now we consider the corresponding two flavor mixing. The states $\nu_{e}$ and $\nu_{\mu}$ are coupled by a Majorana mass term $m_{e \mu}$. Then the mass term in the Lagrangian density is considered as 


$$
\begin{aligned}
(-g)^{-1 / 2} \mathcal{L}_{m} & =-\frac{1}{2}\left(\nu_{e 1}^{\dagger} m_{e 1} \nu_{e 1}+\nu_{e 2}^{\dagger} m_{e 2} \nu_{e 2}+\nu_{\mu 1}^{\dagger} m_{\mu 1} \nu_{\mu 1}+\nu_{\mu 2}^{\dagger} m_{\mu 2} \nu_{\mu 2}\right. \\
& \left.-\nu_{\mu 1}^{\dagger} m_{e \mu} \nu_{e 1}-\nu_{e 1}^{\dagger} m_{e \mu} \nu_{\mu 1}+\nu_{\mu 2}^{\dagger} m_{e \mu} \nu_{e 2}+\nu_{e 2}^{\dagger} m_{e \mu} \nu_{\mu 2}\right)
\end{aligned}
$$

Here we assume that the Majorana mass matrix, coupling $\nu_{e}$ and $\nu_{\mu}$, is Hermitian and diagonal. We also assume that $m_{e \mu}$ in $\nu_{e 1}^{\dagger} \nu_{\mu 1}$ and $\nu_{e 2}^{\dagger} \nu_{\mu 2}$ are same for computational simplicity, while our main goal is to investigate any gravity effect. As our main aim is to study the effect of curvature to the oscillation phase, even for the convenience of transparent understanding of sole effect of gravity we prefer to consider the nongravitating part as simple as possible. This mass term gives rise to two mass matrices $\mathcal{M}_{f(1,2)}$ given by

$$
\mathcal{M}_{f 1}=\left(\begin{array}{cc}
m_{e 1} & -m_{e \mu} \\
-m_{e \mu} & m_{\mu 1}
\end{array}\right), \quad \mathcal{M}_{f 2}=\left(\begin{array}{cc}
m_{e 2} & m_{e \mu} \\
m_{e \mu} & m_{\mu 2}
\end{array}\right)
$$

which mix $\nu_{e 1}\left(\nu_{e 2}\right)$ and $\nu_{\mu 1}\left(\nu_{\mu 2}\right)$. The mixing, leads to two mass eigenstates $f_{11}\left(f_{12}\right)$ and $f_{21}\left(f_{22}\right)$, is expressed as

$$
f=\mathcal{F}^{\dagger} \nu_{f}
$$

where

$$
\nu_{f}=\left(\begin{array}{c}
\nu_{e} \\
\nu_{\mu}
\end{array}\right), \quad f=\left(\begin{array}{c}
f_{1} \\
f_{2}
\end{array}\right)
$$

and $\mathcal{F}$ is given by

$$
\mathcal{F}_{1,2}=\left(\begin{array}{cc}
\cos \phi_{1,2} & -\sin \phi_{1,2} \\
\sin \phi_{1,2} & \cos \phi_{1,2}
\end{array}\right) .
$$

Here the subscript 1 and 2 refer to the flavor mixing between mass eigenstates subscripted by 1 and 2 respectively. Hence

$$
\tan \phi_{1,2}=\frac{\mp 2 m_{e \mu}}{\left(m_{e(1,2)}-m_{\mu(1,2)}\right)+\sqrt{\left(m_{e(1,2)}-m_{\mu(1,2)}\right)^{2}+4 m_{e \mu}^{2}}} .
$$

Thus we obtain all together four mass eigenstates $\chi_{1}, \chi_{2}, \chi_{3}$ and $\chi_{4}$ described as

$$
\left(\begin{array}{l}
\chi_{1} \\
\chi_{2}
\end{array}\right) \equiv\left(\begin{array}{l}
f_{11} \\
f_{21}
\end{array}\right)=\mathcal{F}_{1}^{\dagger}\left(\begin{array}{c}
\nu_{e 1} \\
\nu_{\mu 1}
\end{array}\right) \quad \text { and }\left(\begin{array}{l}
\chi_{3} \\
\chi_{4}
\end{array}\right) \equiv\left(\begin{array}{c}
f_{12} \\
f_{22}
\end{array}\right)=\mathcal{F}_{2}^{\dagger}\left(\begin{array}{c}
\nu_{e 2} \\
\nu_{\mu 2}
\end{array}\right)
$$

with mass eigenvalues $M_{1}, M_{2}, M_{3}$ and $M_{4}$ given by

$$
\begin{aligned}
& M_{1,2} \equiv m_{(1,2) 1}=\frac{1}{2}\left\{\left(m_{e 1}+m_{\mu 1}\right) \pm \sqrt{\left(m_{e 1}-m_{\mu 1}\right)^{2}+4 m_{e \mu}^{2}}\right\} \\
& M_{3,4} \equiv m_{(1,2) 2}=\frac{1}{2}\left\{\left(m_{e 2}+m_{\mu 2}\right) \pm \sqrt{\left(m_{e 2}-m_{\mu 2}\right)^{2}+4 m_{e \mu}^{2}}\right\}
\end{aligned}
$$

Now from Eq. (37)

$$
\begin{array}{r}
m_{e 1}+m_{\mu 1}=-\left(\sqrt{B_{0}^{2}+m_{e}^{2}}+\sqrt{B_{0}^{2}+m_{\mu}^{2}}\right)=-\left(Y_{e}+Y_{\mu}\right) \\
m_{e 2}+m_{\mu 2}=\left(\sqrt{B_{0}^{2}+m_{e}^{2}}+\sqrt{B_{0}^{2}+m_{\mu}^{2}}\right)=Y_{e}+Y_{\mu} \\
m_{e 1}-m_{\mu 1}=-\sqrt{B_{0}^{2}+m_{e}^{2}}+\sqrt{B_{0}^{2}+m_{\mu}^{2}}=-Y_{e}+Y_{\mu} \\
m_{e 2}-m_{\mu 2}=\sqrt{B_{0}^{2}+m_{e}^{2}}-\sqrt{B_{0}^{2}+m_{\mu}^{2}}=Y_{e}-Y_{\mu}
\end{array}
$$


where

$$
Y=\sqrt{B_{0}^{2}+m^{2}}
$$

If we define

$$
2 m_{a}=Y_{\mu}+Y_{e}, \quad 2 m_{i}=Y_{\mu}-Y_{e}
$$

then

$$
\begin{gathered}
m_{e 1}+m_{\mu 1}=-2 m_{a}, \quad m_{e 1}-m_{\mu 1}=2 m_{i} \\
m_{e 2}+m_{\mu 2}=2 m_{a}, \quad m_{e 2}-m_{\mu 2}=-2 m_{i} .
\end{gathered}
$$

Then we have

$$
\begin{array}{r}
M_{1}=-m_{a}+\sqrt{m_{i}^{2}+m_{e \mu}^{2}}, \quad M_{2}=-m_{a}-\sqrt{m_{i}^{2}+m_{e \mu}^{2}}, \\
M_{3}=m_{a}+\sqrt{m_{i}^{2}+m_{e \mu}^{2}}, \quad M_{4}=m_{a}-\sqrt{m_{i}^{2}+m_{e \mu}^{2}}
\end{array}
$$

and

$$
\tan \phi_{1,2}=\frac{\mp m_{e \mu}}{ \pm m_{i}+\sqrt{m_{i}^{2}+m_{e \mu}^{2}}}
$$

Note that at $B_{0}=0$ all the above results reduce to that of conventional two flavor mixing without gravity effect. For example, at $B_{0}=0$

$$
\begin{aligned}
& \psi_{e, \mu}=\frac{1}{\sqrt{2}}\left(\nu_{(e, \mu) 1}+\nu_{(e, \mu) 2}\right), \\
& \psi_{e, \mu}^{c}=\frac{1}{\sqrt{2}}\left(\nu_{(e, \mu) 1}-\nu_{(e, \mu) 2}\right) .
\end{aligned}
$$

Then substituting $\nu$-s by $\psi$-s in Eq. (39) with $m_{(e, \mu) 1}=-m_{e, \mu}$ and $m_{(e, \mu) 2}=m_{e, \mu}$ one obtains the standard mass Lagrangian density with spinors $\psi$ and $\psi^{c}[25]$

$$
\mathcal{L}_{m}=-\frac{1}{2}\left(\psi_{e}^{c \dagger} m_{e} \psi_{e}+\psi_{e}^{c \dagger} m_{e \mu} \psi_{\mu}+\psi_{\mu}^{c \dagger} m_{e \mu} \psi_{e}+\psi_{\mu}^{c \dagger} m_{\mu} \psi_{\mu}\right)+\text { h.c. }
$$

The readers comparing this result with that in 25] should not confuse with difference in notation in the present paper from that in [25]. Here $\nu_{e, \mu}$ are the Majorana neutrino fields of electron and muon typed under gravity whose oscillation would be interesting to study.

The most general flavor mixing mass matrix in presence of gravity is given by

$$
\mathcal{M}_{4}=\left(\begin{array}{cc}
-B_{0} \mathbf{I} & -\mathbf{M} \\
-\mathbf{M} & B_{0} \mathbf{I}
\end{array}\right)
$$

when $\mathbf{I}$ is the $2 \times 2$ unit matrix and

$$
\mathbf{M}=\left(\begin{array}{cc}
m_{e} & m_{e \mu} \\
m_{e \mu} & m_{\mu}
\end{array}\right) \equiv \mathbf{U}_{\theta} \cdot \operatorname{diag}\left(m_{1}, m_{2}\right) \cdot \mathbf{U}_{\theta}^{\dagger},
$$

where $\mathbf{U}_{\theta}$ and $m_{1,2}$ are the mixing matrix in vacuum and the neutrino masses respectively in absence of curvature. The the corresponding flavor state is given by

$$
\Psi=\left(\begin{array}{c}
\psi_{e}^{c} \\
\psi_{\mu}^{c} \\
\psi_{e} \\
\psi_{\mu}
\end{array}\right)
$$

However, for the sake of simplicity we take an assumption such that the $4 \times 4$ mass matrix is resolved into $2 \times 2$ block form. The motivation is to study the gravity effect in a transparent manner. In order to do that we consider 
the mixing part by part as described above: first we take the gravity effect on the flavor eigenstates and then we mix them up. The argument for this assumption is as follows. We detect neutrinos by weak interactions. In standard experiments, we detect and produce them via charge-current interaction. As the flavor eigenstates take part in charge-current interaction, we detect and produce them only. In our calculation, the original flavor eigenstates are $\psi$ and $\psi^{c}$ or as a whole $\Psi$. Therefore, we plan to discuss the oscillation probability and corresponding length among states $\Psi$. However, when we consider them under strong gravity, they couple and no longer have been a definite mass state, rather modify to a state $\nu$. Hence it is worthwhile to study the oscillation properties for states $\nu$ as well. Indeed the solution of the Dirac equation in presence of gravitational interaction is different than that in absence of gravity. Hence while performing the oscillation experiment under gravity, it can be assumed that gravity will affect the initially produced flavor states and in between the detection and production the flavor mixing Hamiltonian will act upon these gravitationally modified flavor states. Therefore, even though they are produced as flavor eigenstates $\Psi$ via weak interaction, if system is under strong gravity, then they are modified to $\nu$, as described in $\S I I I . A, B$, which may be observable in presence of strong curvature. Indeed $\nu \equiv \Psi$ when $B_{0}=0$. If we put $B_{0}=0$ in our oscillation probability and oscillation length, described in $\S I V . B$ below, then we obtain the standard expressions of those in flat space. This validates our assumption of mixing scheme and verifies that our results for $\nu$ are physically same as that for $\Psi$ under gravity, as will be discussed in $§ I V . C$.

\section{B. Flavor oscillation}

The sets of two Majorana neutrino flavor eigenstates are described as

$$
\begin{aligned}
& \left|\nu_{e 1}\right\rangle=\cos \phi_{1}\left|\chi_{1}\right\rangle-\sin \phi_{1}\left|\chi_{2}\right\rangle \\
& \left|\nu_{\mu 1}\right\rangle=\sin \phi_{1}\left|\chi_{1}\right\rangle+\cos \phi_{1}\left|\chi_{2}\right\rangle
\end{aligned}
$$

and

$$
\begin{gathered}
\left|\nu_{e 2}\right\rangle=\cos \phi_{2}\left|\chi_{3}\right\rangle-\sin \phi_{2}\left|\chi_{4}\right\rangle \\
\left|\nu_{\mu 2}\right\rangle=\sin \phi_{2}\left|\chi_{3}\right\rangle+\cos \phi_{2}\left|\chi_{4}\right\rangle .
\end{gathered}
$$

Let us now consider the states given by Eqs. (58). The oscillation probability of those states is

$$
\mathcal{P}_{f g 1}=\sin ^{2} 2 \phi_{1} \sin ^{2} \delta_{f g 1}(t),
$$

where

$$
\delta_{f g 1}(t)=\frac{\left|M_{1}^{2}-M_{2}^{2}\right|}{4 E} t
$$

and $E$ is the energy of the mass eigenstates. Hence the oscillation length is

$$
\lambda_{f g 1}=\frac{4 \pi E}{\left|M_{1}^{2}-M_{2}^{2}\right|} .
$$

Similarly, for the states of Eqs. (59) the oscillation probability is

$$
\mathcal{P}_{f g 2}=\sin ^{2} 2 \phi_{2} \sin ^{2} \delta_{f g 2}(t),
$$

where

$$
\delta_{f g 2}(t)=\frac{\left|M_{3}^{2}-M_{4}^{2}\right|}{4 E} t
$$

and the oscillation length is

$$
\lambda_{f g 2}=\frac{4 \pi E}{\left|M_{3}^{2}-M_{4}^{2}\right|} .
$$

Now from Eqs. (47), (49) and (51) we obtain

$$
\begin{array}{r}
\Delta M^{2}=\left|M_{1}^{2}-M_{2}^{2}\right|=\left|M_{3}^{2}-M_{4}^{2}\right| \\
=\left(\sqrt{B_{0}^{2}+m_{\mu}^{2}}+\sqrt{B_{0}^{2}+m_{e}^{2}}\right) \\
\times \sqrt{\left\{\left(\sqrt{B_{0}^{2}+m_{\mu}^{2}}-\sqrt{B_{0}^{2}+m_{e}^{2}}\right)^{2}+4 m_{e \mu}^{2}\right\}} .
\end{array}
$$


From Eq. (52), we obtain

$$
\sin ^{2} 2 \phi_{1}=\sin ^{2} 2 \phi_{2}=\sin ^{2} 2 \phi=\frac{m_{e \mu}^{2}}{m_{i}^{2}+m_{e \mu}^{2}}
$$

so that the oscillation probability and the oscillation length are same for the two cases respectively given by

$$
\begin{gathered}
\mathcal{P}_{f g}=\sin ^{2} 2 \phi \sin ^{2} \delta_{f g}(t), \\
\lambda_{f g}=\frac{4 \pi E}{\Delta M^{2}} .
\end{gathered}
$$

However, $\Psi$-s are related to $\nu$-s. Hence once we obtain the oscillation probability and length between $\nu_{e 1}$ (or $\nu_{e 2}$ ) and $\nu_{\mu 1}\left(\right.$ or $\left.\nu_{\mu 2}\right)$, the results can be converted to that for $\psi_{e}$ and $\psi_{\mu}$ given in the next subsection.

\section{Oscillation probability between initial flavors produced via weak interaction}

It is evident from Eqs. (35) and (41) that we can express the initial flavor states $\Psi_{e}$ and $\Psi_{\mu}$ in terms of mass eigenstates $\chi$-s given by

$$
\begin{gathered}
\psi_{e}^{c}=\cos \theta_{e} \cos \phi_{1} \chi_{1}-\cos \theta_{e} \sin \phi_{1} \chi_{2}-\sin \theta_{e} \cos \phi_{2} \chi_{3}+\sin \theta_{e} \sin \phi_{2} \chi_{4}, \\
\psi_{\mu}^{c}=\cos \theta_{\mu} \sin \phi_{1} \chi_{1}+\cos \theta_{\mu} \cos \phi_{1} \chi_{2}-\sin \theta_{\mu} \sin \phi_{2} \chi_{3}-\sin \theta_{\mu} \cos \phi_{2} \chi_{4}, \\
\psi_{e}=\sin \theta_{e} \cos \phi_{1} \chi_{1}-\sin \theta_{e} \sin \phi_{1} \chi_{2}+\cos \theta_{e} \cos \phi_{2} \chi_{3}-\cos \theta_{e} \sin \phi_{2} \chi_{4}, \\
\psi_{\mu}=\sin \theta_{\mu} \sin \phi_{1} \chi_{1}+\sin \theta_{\mu} \cos \phi_{1} \chi_{2}+\cos \theta_{\mu} \sin \phi_{2} \chi_{3}+\cos \theta_{\mu} \cos \phi_{2} \chi_{4} .
\end{gathered}
$$

In short, we can write this as

$$
\left(\begin{array}{l}
\psi_{e}^{c} \\
\psi_{\mu}^{c} \\
\psi_{e} \\
\psi_{\mu}
\end{array}\right)=T\left(\begin{array}{l}
\chi_{1} \\
\chi_{2} \\
\chi_{3} \\
\chi_{4}
\end{array}\right)
$$

where

$$
T=\left(\begin{array}{cccc}
\cos \theta_{e} \cos \phi_{1} & -\cos \theta_{e} \sin \phi_{1} & -\sin \theta_{e} \cos \phi_{2} & \sin \theta_{e} \sin \phi_{2} \\
\cos \theta_{\mu} \sin \phi_{1} & \cos \theta_{\mu} \cos \phi_{1} & -\sin \theta_{\mu} \sin \phi_{2} & -\sin \theta_{\mu} \cos \phi_{2} \\
\sin \theta_{e} \cos \phi_{1} & -\sin \theta_{e} \sin \phi_{1} & \cos \theta_{e} \cos \phi_{2} & -\cos \theta_{e} \sin \phi_{2} \\
\sin \theta_{\mu} \sin \phi_{1} & \sin \theta_{\mu} \cos \phi_{1} & \cos \theta_{\mu} \sin \phi_{2} & \cos \theta_{\mu} \cos \phi_{2}
\end{array}\right) .
$$

From this the particle part $\psi$ can be written as

$$
\begin{gathered}
\psi_{e}=T_{e 1} \chi_{1}+T_{e 2} \chi_{2}+T_{e 3} \chi_{3}+T_{e 4} \chi_{4}, \\
\psi_{\mu}=T_{\mu 1} \chi_{1}+T_{\mu 2} \chi_{2}+T_{\mu 3} \chi_{3}+T_{\mu 4} \chi_{4} .
\end{gathered}
$$

Hence we compute the oscillation probability between $\psi_{e}$ and $\psi_{\mu}$

$$
\mathcal{P}_{i g}=-4 \sum_{i<j=1}^{4} T_{e i} T_{e j} T_{\mu i} T_{\mu j} \sin ^{2} \delta_{i j}(t)
$$

where

$$
\delta_{i j}(t)=\frac{\left|\Delta M_{i j}^{2}\right|}{4 E} t, \quad \Delta M_{i j}^{2}=M_{i}^{2}-M_{j}^{2} .
$$

This resembles the oscillation probability one obtains in the case of 3 flavors mixing or in general the case of $N$ flavors mixing. With actual expressions of $T_{(e, \mu) i}$-s from Eq. (70) we obtain

$$
\begin{gathered}
\mathcal{P}_{i g}=\sin ^{2} \theta_{e} \sin ^{2} \theta_{\mu} \sin ^{2} 2 \phi_{1} \sin ^{2} \delta_{12}(t)+\cos ^{2} \theta_{e} \cos ^{2} \theta_{\mu} \sin ^{2} 2 \phi_{2} \sin ^{2} \delta_{34}(t) \\
-\frac{1}{4} \sin 2 \theta_{e} \sin 2 \theta_{\mu} \sin 2 \phi_{1} \sin 2 \phi_{2}\left\{\sin ^{2} \delta_{13}(t)-\sin ^{2} \delta_{14}(t)-\sin ^{2} \delta_{23} t+\sin ^{2} \delta_{24}(t)\right\} .
\end{gathered}
$$


From Eq. (51)

$$
\left|M_{1}^{2}-M_{3}^{2}\right|=\left|M_{2}^{2}-M_{4}^{2}\right|=\Delta M^{2}=4 m_{a} \sqrt{m_{i}^{2}+m_{e \mu}^{2}}
$$

and

$$
\left|M_{1}^{2}-M_{4}^{2}\right|=\left|M_{2}^{2}-M_{3}^{2}\right|=0
$$

Moreover, from Eq. (52)

$$
\sin 2 \phi_{1}=-\sin 2 \phi_{2}=\sin 2 \phi \text { (say). }
$$

Hence the oscillation probability is given by

$$
\mathcal{P}_{i g}=\sin ^{2} 2 \phi\left\{\left(\sin \theta_{e} \sin \theta_{\mu}+\cos \theta_{e} \cos \theta_{\mu}\right)^{2} \sin ^{2}\left(\frac{\Delta M^{2}}{4 E} t\right)\right\} .
$$

Therefore, we find that the oscillation length between $\psi$ states is same as that in the case of oscillation between $\nu_{1}$ (and $\left.\nu_{2}\right)$ states: equivalent to each other.

Now the oscillation length would change for a distant observer due to gravitational redshift. Therefore, the redshifted oscillation length

$$
\lambda_{f r}=\frac{\lambda_{f}}{\sqrt{g_{t t}}}
$$

If we consider the neutrino flavor oscillation in a rotating black hole spacetime of mass $M$ and specific angular momentum $a$, then at a point $(r, \theta)$

$$
g_{t t}=1-\frac{2 M r}{\rho^{2}}
$$

with

$$
\rho^{2}=r^{2}+a^{2} \cos \theta
$$

Therefore, it is evident from the above discussions that under gravity the probability of flavor oscillation changes significantly depending on the gravitational strength.

We now consider Majorana mass of electron neutrino $m_{e} \sim 5 \times 10^{-3} \mathrm{eV}$, muon neutrino $m_{\mu} \sim 6.5 \times 10^{-3} \mathrm{eV}$ and mixing $m_{e \mu} \sim 3.5 \times 10^{-3} \mathrm{eV}$. These values are consistent with the solar neutrino oscillation data. Thus, without gravity the oscillation probability between electron and muon neutrino with $\Delta M^{2}\left(B_{0}=0\right)=8.2 \times 10^{-5} \mathrm{eV}^{2}$, as from solar neutrino data [26], is given by

$$
\mathcal{P}_{f}=\mathcal{P}_{f g}\left(B^{0}=0\right) \simeq 0.956 \sin ^{2}\left(\frac{8.2 \times 10^{-5} \mathrm{eV}^{2}}{4 E} t\right)
$$

The corresponding oscillation length

$$
\lambda_{f}=\lambda_{f g}\left(B_{0}=0\right) \simeq \frac{4 \pi E}{8.2 \times 10^{-5} \mathrm{eV}^{2}}
$$

\section{Oscillation around black holes}

We first recall the gravitational scalar potential computed earlier in the Kerr geometry [14]

$$
B^{0}=-\frac{4 a \sqrt{M} z}{\bar{\rho}^{2} \sqrt{2 r^{3}}}
$$

for a black hole of mass $M$ and specific angular momentum $a$, where $\bar{\rho}^{2}=2 r^{2}+a^{2}-x^{2}-y^{2}-z^{2}$. If we consider neutrinos at around 20 Schwarzschild radius in the spacetime of a primordial black hole of mass $10^{22} \mathrm{gm}$, then from 
Eq. (86) the gravitational field is computed to be $\sim 10^{-2} \mathrm{eV}$ which is comparable to neutrino masses. If we specify the gravitational field $B_{0} \sim 5 \times 10^{-2} \mathrm{eV}$, then from Eqs. (68) the oscillation probability becomes

$$
\mathcal{P}_{f B H} \simeq 0.999 \sin ^{2}\left(\frac{7 \times 10^{-4} \mathrm{eV}^{2}}{4 E} t\right) \text {. }
$$

The oscillation length, from Eq. (69), is determined as

$$
\lambda_{f B H} \simeq \frac{4 \pi E}{7 \times 10^{-4} \mathrm{eV}^{2}} .
$$

Hence, the oscillation length decreases by almost an order of magnitude.

If $B_{0}$ is much higher than neutrino Majorana masses, then from the Eq. (67) we have $\sin ^{2} 2 \phi \sim 1$ and $\Delta M^{2} \sim$ $4 m_{e \mu} B_{0}$. Therefore, the oscillation probability

$$
\mathcal{P}_{f B H}=\sin ^{2}\left(\frac{1.4 \times 10^{-2} \mathrm{eVB} B_{0}}{4 E} t\right)
$$

and the oscillation length

$$
\lambda_{f B H} \simeq \frac{4 \pi E}{1.4 \times 10^{-2} \mathrm{eVB} B_{0}} .
$$

\section{E. Oscillation in early universe}

\section{Anisotropic universe}

We recall the gravitational scalar potential in the anisotropic phase of early universe [13, 14]

$$
B^{0}=\frac{4 R^{3} S+3 y^{2} R S^{3}-2 y S^{4}}{8 R^{4}+2 y^{2} R^{2} S^{2}} .
$$

If we consider radiation dominated era with $R(t)=\left(t / t_{0}\right)^{1 / 2}$ and $S(t)$ as an arbitrary constant $\geq 1$, then the above potential reduces to

$$
B_{0} \sim \frac{S^{2}}{y}\left(\frac{t_{0}}{t}\right)
$$

when $y$ is the position coordinate, can not be greater than the size of universe of corresponding era, and $t_{0}$ is the present age of universe $\sim 10^{17} \mathrm{sec}$. At the neutrino decoupling age of universe when $t \sim 1 \mathrm{sec}, y \leq 10^{20} \mathrm{~cm}, B_{0} \geq 10^{-8}$ $\mathrm{eV}$. Therefore, if $B_{0} \sim 5 \times 10^{-2} \mathrm{eV}$, then from Eqs. (68) and (87) the oscillation probability is given by

$$
\mathcal{P}_{f E U_{d}} \leq 0.999 \sin ^{2}\left(\frac{7 \times 10^{-4} \mathrm{eV}^{2} \mathrm{sec}}{4 E}\right)
$$

On the other hand, at GUT scale when $t \sim 10^{-35} \mathrm{sec}, y \leq 10^{2} \mathrm{~cm}, B_{0} \geq 10^{45} \mathrm{eV}>>m_{e}, m_{\mu}, m_{e \mu}$. Therefore, the oscillation probability with the minimum possible $B_{0}$

$$
\mathcal{P}_{f E U_{G U T}} \leq \sin ^{2}\left(\frac{1.4 \times 10^{8} \mathrm{eV}^{2} \mathrm{sec}}{4 E}\right)
$$

is entirely controlled by gravitational field. Either of Eqs. (93) and (94) clearly proves that flavor oscillation is severely altered by the gravity.

\section{Inflationary era of universe with primordial fluctuations}

We know that during inflation primordial quantum fluctuations of the spacetime is classical and the flat RobertsonWalker expanding universe may take the form as [27, 28] 


$$
d s^{2}=(1+2 \tilde{\phi}) d t^{2}-a(t)^{2}\left[\frac{\omega_{i}}{a(t)} d x^{i} d t+\left((1+2 \tilde{\psi}) \delta_{i j}+h_{i j}\right) d x^{i} d x^{j}\right]
$$

where $\tilde{\phi}$ and $\tilde{\psi}$ are scalar, $\omega_{i}$ are vector and $h_{i j}$ are the tensor fluctuations of the metric. Of the ten degrees of freedom in the metric perturbations only six are independent and the remaining four can be set to zero by suitable gauge choice. For our application we must have atleast one nonzero $g_{i j}$, when $i \neq j=1,2,3$, of the metric [10] and thus need only the tensor perturbations and we choose the transverse-traceless (TT) gauge $h_{i}^{i}=0, \partial^{i} h_{i j}=0$ for the tensor perturbations. In the TT gauge the above perturbed Robertson-Walker metric can be expressed as

$$
\begin{array}{r}
d s^{2}=(1+2 \tilde{\phi}) d t^{2}-a(t)^{2}\left[\frac{\omega_{i}}{a(t)} d x^{i} d t+\left(1+2 \tilde{\psi}-h_{+}\right) d x_{1}^{2}+\left(1+2 \tilde{\psi}+h_{+}\right) d x_{2}^{2}+\right. \\
\left.2 h_{\times} d x_{1} d x_{2}+(1+2 \tilde{\psi}) d x_{3}^{2}\right] .
\end{array}
$$

Therefore, following general expression [10, 14] given for any metric the gravitational scalar potential computed for this spacetime is

$$
B^{0}=\partial_{3} h_{\times}=\partial_{z} h_{\times}
$$

The gravitational scalar potential $B_{0}$ can be expressed as a fluctuation amplitude $A_{\times}$times a wavenumber which represents the length scale over which the metric fluctuations vary. The Compton wavelength of the particles in the GUT era is much smaller than the average wavelength of the gravitational waves whose wavenumber $k \sim H=$ $1.66 g_{*}^{1 / 2}\left(T^{2} / M_{P l}\right)[27,28] . H$ is the Hubble constant at the time of horizon crossing of mode $k$. Thus gravitational wave background can be considered as a constant amplitude field for the GUT processes. Hence the mean value of $B_{0}$, as a function of temperature and the primordial tensor wave amplitude $A_{\times}$, can be expressed as

$$
\left\langle B_{0}\right\rangle \equiv B_{0} \simeq A_{\times} k \simeq A_{\times}\left(1.66 g_{*}^{1 / 2} \frac{T^{2}}{M_{P l}}\right)
$$

Here $g_{*}=106.7$, is the number of relativistic degrees of freedom, for the standard model. Primordial tensor and scalar perturbations contribute to the anisotropy of cosmic microwave background at large angles. The COBE DMR measurement [29] of temperature anisotropy $\Delta T=30 \mu K$ sets an upper limit of these fluctuation amplitudes to be $10^{-5}$. The magnitude of the tensor perturbations depends upon the details of inflation potential [30] and is expected to be an order of magnitude smaller in amplitude than scalar perturbations. Therefore, we can set $A_{\times} \leq 10^{-6}$. Hence, at $T \sim 10^{13} \mathrm{GeV}$ with a very small amplitude of fluctuation such that $A_{\times} \sim 10^{-19}$ the gravitational potential comes out to be $B_{0} \sim 10^{-2} \mathrm{eV}$ which is only an order of magnitude higher than the neutrino masses. Therefore, the corresponding oscillation probability and length are given by

$$
\begin{aligned}
& \mathcal{P}_{\text {fIN }}=0.999 \sin ^{2}\left(\frac{7 \times 10^{-35} \mathrm{eV}^{2} \mathrm{sec}}{4 E}\right), \\
& \lambda_{\text {fIN }} \simeq \frac{4 \pi E}{7 \times 10^{-4} \mathrm{eV}^{2}}
\end{aligned}
$$

which are an order of magnitude higher than that without gravitational effect. At the maximum possible $A_{\times}$, $B_{0} \sim 10^{11} \mathrm{eV}>>m_{e}, m_{\mu}, m_{e \mu}$ resulting the oscillation probability and length

$$
\begin{aligned}
& \mathcal{P}_{f I N}=\sin ^{2}\left(\frac{1.4 \times 10^{-22} \mathrm{eV}^{2} \mathrm{sec}}{4 E}\right), \\
& \lambda_{f I N} \simeq \frac{4 \pi E}{1.4 \times 10^{-2} \mathrm{eV} \mathrm{B} B_{0}} .
\end{aligned}
$$

\section{F. Implications}

The flavor mixing and hence oscillation is influenced by gravity. This may alter the relative abundance of different neutrino flavors in universe. Around a primordial black hole the maximum oscillation length of a thermal neutrino, from Eqs. (88) and (90), is obtained as $0.54 \mathrm{~cm}$ compared to $4.6 \mathrm{~cm}$ obtained from Eq. (85) without gravity. 
The oscillation probability increases as well significantly in early universe when we consider gravitational effect, as understood from section IV.D. At the neutrino decoupling era, while the probability increases for thermal neutrinos only 1.5 times, for $\mathrm{TeV}$ neutrinos it increases about two order of magnitude. It is also apparent from Eqs. (84), (93) and (94) that at GUT era oscillation takes place vigorously. Therefore, production of muon neutrinos in early universe due to oscillation is expected to be much higher than that estimated without gravitational effect.

At the inflationary era with primordial fluctuations, the oscillation length of, e.g., GeV neutrinos could vary from $10^{8} \mathrm{~cm}$ to $10^{-5} \mathrm{~cm}$ depending on $B_{0}$, while size of universe is $10^{3} \mathrm{~cm}$. Therefore, the oscillation would be feasible. However, lifetime of the era itself is very small which may hinder significant oscillation.

\section{NEUTRINOLESS DOUBLE BETA DECAY}

It is generally believed that if neutrino is its own antiparticle, i.e. neutrino is a Majorana particle, then neutrinoless double beta decay may be observed. As CPT violating nature alters the mixing angle and masses in neutrino and antineutrino sector, it is interesting to see the effect of gravity on neutrinoless double beta decay rate. If one considers only one flavor, then gravity does not alter the decay rate. It was already shown 7] that CPT violating term has no effect on decay rate with only one flavor. Here we investigate the case for two flavor mixing.

If we consider neutrino flavor mixing, without neutrino-antineutrino mixing, then one can express neutrino of different flavors as superposition of different mass eigenstates like (considering only electron and muon neutrino)

$$
\left(\begin{array}{l}
\psi_{e} \\
\psi_{\mu}
\end{array}\right)=U\left(\begin{array}{l}
f_{1} \\
f_{2}
\end{array}\right)
$$

where $U=\mathcal{F}\left(B^{0}=0\right)$, and $f_{1}$ and $f_{2}$ are mass eigenstates with masses $l_{1}$ and $l_{2}$ respectively. In this case, the amplitude for neutrinoless double beta decay involving only electrons is [31]

$$
A \propto \sum_{i} l_{i} U_{e i}^{2}, \quad i=1,2 .
$$

In presence of gravity the state $\Psi$ is replaced by $\nu$ as discussed in previous sections, and so the relation given by the Eq. (101) is modified to

$$
\left(\begin{array}{c}
\nu_{e} \\
\nu_{\mu}
\end{array}\right)=\mathcal{F}\left(\begin{array}{l}
f_{1} \\
f_{2}
\end{array}\right)
$$

Hence, the neutrinoless double beta decay amplitude is given by

$$
A_{(1,2)} \propto M_{(1,3)} \cos ^{2} \phi_{(1,2)}+M_{(2,4)} \sin ^{2} \phi_{(1,2)} .
$$

From the expressions of $M$-s and $\phi$-s from Eqs. (51) and (52) and using Eqs. (48) and (49) we obtain

$$
A \propto \sqrt{B_{0}^{2}+m_{e}^{2}} .
$$

Hence, under the effect of gravity the neutrinoless double beta decay amplitude is modified as the mass of the flavor state is modified. In absence of gravity the amplitude is simply proportional to $m_{e}$.

The double beta decay is involved with weak interactions, conventionally it has nothing to do gravity. Therefore, when we compute the decay rate with $\Psi$-s, which are the states produced by pure charge-current interaction, it remains unchanged in presence of gravity. However, if we assume that under strong gravity $\Psi$-s modify to $\nu$-s and recalculate the rate, then it modifies by means of $B_{0}$.

\section{SUMMARY AND DISCUSSION}

The idea of neutrino flavor oscillation is well established both theoretically and experimentally. However, there are some anomalies in different types of flavor oscillation data. For example, the LSND data provides mixing angle and neutrino masses which are inconsistent with solar and atmospheric neutrino oscillation data. This anomaly can be removed by introducing fourth family of sterile neutrino or CPT violation in neutrino sector. In a very past work, neutrino-antineutrino oscillation was discussed in analogy with $K^{0}-\bar{K}^{0}$ oscillation with maximal mixing [32]. In the 
present paper, we have shown that in presence of gravity neutrino-antineutrino oscillation does occur. Neutrino and antineutrino interact differently with gravity through CPT violation. Under this condition if the Lagrangian has a lepton number violating Majorana mass, then neutrino-antineutrino mixing and oscillation occur. Without gravity, Majorana mass mixes neutrino and antineutrino states in equal ratio. Even if there is no Majorana mass, gravity splits neutrino and antineutrino in two different mass eigenstates. The gravitational scalar potential $B_{0}$ modifies the mass of the neutrino states. This $B_{0}$ behaves like a lepton number conserving mass. The mass eigenstates, produced as a result of mixing under gravity, act as modified neutrino states. With these states we have studied two flavor mixing and corresponding oscillation generating four mass eigenstates.

It is quite possible that the neutrino number what we see today is carried out off the neutrino decoupling era and the oscillation probability, strongly influenced by gravity, at that era should determine the relative abundance of today's flavors. We, in fact, have shown that the probability of conversion of electron neutrino to muon neutrino has greatly been enhanced, upto a few order of magnitude, with inclusion of gravity effect. On the other hand, even in present age the relative abundance of muon neutrino may be increased around primordial black holes where gravitational field is not negligible.

It has been suggested that during core-collapse of a massive star, part of the infalling material goes into orbit around the compact core to form a hot, dense, centrifugally supported accretion disk whose evolution is strongly influenced by neutrino interactions. Under a wide range of conditions, this neutrino-dominated accretion flow will help to produce a successful supernova explosion (see, e.g. [34]) It will be interesting to study the effect of gravity on oscillation in such systems and then on related supernova explosion.

In early, it was shown by pure quantum field theoretical consideration of neutrino flavor mixing that in infinite volume limit, the vacuum expectation value is not invariant under the transformation of flavor mixing (e.g. [35, 36]). This is the consequence of unitary inequivalance of the flavor and mass vacua. It has been shown that the vacuum structure with neutrino mixing has non-zero contribution to vacuum energy [37]. It is well known that one of the interpretations of cosmological constant is linked with the density $(\rho)$ and pressure $(p)$ of vacuum with equation of state $p=-\rho$. The non-zero value of cosmological constant and hence the presence of such a vacuum energy density, which is often termed as dark energy, is needed to explain the observed acceleration of present Universe. Thus, the non-zero flavor vacuum energy density can be interpreted as a contribution to cosmological constant and hence to dark energy [35]. It has been shown [35] that the vacuum energy, the flavor vacuum expectation value of energy momentum tensor element $T_{00}$, is non-zero and proportional to square of one of the Bogoliubov coefficients $V_{k}$ associated with the flavor creation and annihilation operator. In ultrarelativistic limit $\left|V_{k}\right|^{2}$ is proportional to $\left(m_{1}-m_{2}\right)^{2}$, the square of the difference of mass eigenvalues. Moreover, this vacuum expectation value is proportional to $\sin ^{2} \phi$, where $\phi$ is the mixing angle. Hence this contribution depends on the specific nature of the mixing. It is to be noted that in the limit $V_{k} \rightarrow 0$, which is the case of traditional phenomenological mixing, the vacuum energy vanishes. All these calculations are in flat space. If we consider any curved spacetime, then the vacuum expectation value changes accordingly. Hence the contribution to vacuum density and then to dark energy depends on specific nature of mixing and background metric. In the present work, we have shown that background curvature i.e the gravitational field affects the mixing. Hence, in this case, we expect that gravity will play in both the ways - by affecting the mixing and by giving a curved background, to affect the contribution of dark energy. Therefore, it will be good to see how the present results can account for observed proportion of dark energy under the pure quantum field theoretic consideration.

With this new mixing scheme, neutrinoless double beta decay rate has been revisited. With one neutrino flavor, it was shown [7] earlier that CPT violating term has no effect on decay rate. In this spirit we have calculated the amplitude of neutrinoless double beta decay considering neutrino-antineutrino admixture with two neutrino flavor mixing and still have found that the decay amplitude is proportional to the mass of the flavor state. However, as the mass of the flavor state, which is considered to be modified due to gravity, is now different from that in absence of gravity, the decay amplitude also differs from that in absence of gravity. Hence, the CPT violating gravity has an effect on the decay rate.

\section{Acknowledgments}

The authors are grateful to Palash B. Pal of SINP for illuminating discussion throughout the course of the work. They are also thankful to Sudhir K. Vempati of IISc for discussion while writing the paper. The authors would also like to thank the referee for his/her illuminating suggestions and encouragement.

[1] M. C. Gonzalez-Garcia and Y. Nir, Rev. Mod. Phys. 75, 345 (2003). 
[2] G. Barenboim, L. Borissov, J. Lykken and A. Y. Smirnov, JHEP 0210, 001 (2002).

[3] G. Barenboim, L. Borissov and J. Lykken, Phys. Lett. B534, 106 (2002).

[4] H. Murayama and T. Yanagida, Phys. Lett. B520, 253 (2001).

[5] See, http: //www.fnal.gov/pub/presspass/press_releases /BooNE-box.html

[6] V. D. Barger, S. Pakvasa, T. J. Weiler and K. Whisnant, Phys. Rev. Lett. 85, 5055 (2000).

[7] G. Barenboim, J. F. Beacom, L. Borissov and B. Kayser, Phys. Lett. B537, 227 (2002).

[8] S. Mohanty, B Mukhopadhyay and A. R. Prasanna, Phys. Rev. D 65, 122001 (2002).

[9] P. Singh and B Mukhopadhyay, Mod. Phys. Lett. A18, 779 (2003).

[10] B. Mukhopadhyay, Mod. Phys. Lett. A20, 2145 (2005).

[11] D. V. Ahluwalia-Khalilova and D. Grumiller, Phys. Rev. D72, 0067701 (2005)

[12] D. V. Ahluwalia-Khalilova and D. Grumiller, JCAP 07, 012 (2005)

[13] U. Debnath, B. Mukhopadhyay and N. Dadhich, Mod. Phys. Lett. A21, 399 (2006).

[14] B. Mukhopdhyay, Class. Quantum Grav. 24, 1433 (2007).

[15] L. Parker, Phys. Rev. Lett. 44 (1980) 1559; Phys. Rev. D22, 1922 (1980).

[16] F. W. Hehl and W. T. Ni, Phys. Rev. D42, 2045 (1990).

[17] E. Fischbach, B. S. Freeman and W. K. Cheng, Phys. Rev. D23, 2157 (1981).

[18] D. Choudhary, N. D. Hari Dass and M. V. N. Murthy, Class. Quantum Grav. 6, L167 (1989).

[19] C. Q. Xia and Y. L. Wu, Phys. Lett. A141, 251 (1989).

[20] Z. Lalak, S. Pokorski and J. Wess, Phys. Lett. B355, 453 (1995).

[21] J. F. Nieves and and P. B. Pal, Phys. Rev D58, 096005 (1998).

[22] S. Wajima, M. Kasai and T. Futamase, Phys. Rev. D55, 1964 (1999).

[23] K. Varju and L. H. Ryder, Phys. Rev. D62, 024016 (2000).

[24] D. Colladay and V. A. Kostelecký, Phys. Rev. D55, 6760 (1997).

[25] S. M. Bilenky and B. Pontecorvo, Phys. Rep. 41, 225 (1978).

[26] A. Strumia and F. Vissani, hep-ph/0606054

[27] P. J. E. Peebles, Principles of Physical Cosmology, Princeton Univ. Press, Pinceton, NJ (1993);

A. R. Liddle and D. H. Lyth Cosmological Inflation and Large-Scale structure, Cambridge Univ Press, (2000).

[28] S. Mohanty, B. Mukhopadhyay and A. R. Prasanna, hep-ph/0204257.

[29] G. Smoot et al., Astroph. J. 396, L1 (1992);

E. L. Wright Astroph J. 396 , L3 (1992).

[30] V. A. Rubakov, M. Sazhin and A. Veryaskin, Phys. Lett. B 115, 189 (1989);

R. Fabbri and M. Pollock, Phys. Lett. B 125, 445 (1983);

L. Abbot and M. Wise, Nucl. Phys. B 244, 541 (1984);

B. Allen, Phys. Rev. D 37 (1988); L. P. Grischuk, Phys. Rev. Lett. 70, 2371 (1993);

M. S. Turner, Phys. Rev. D48, 3502 (1993);

M. S. Turner, M. White, J. E. Lidsey, Phys. Rev. D48, 4613 (1993).

[31] M. Doi, T. Kotani, H. Nishiura, K. Okuda, \& E. Takasugi, Phys. Lett. B102, 323 (1981).

[32] B. Gribov and V. Pontecorvo, Phys. Lett. B28, 493 (1969).

[33] S. L. Shapiro and S. A. Teukolsky, in Black Holes, White Dwarfs, and Neutron Stars: The Physics of Compact Objects (New York: John Wiley \& Sons) (1983).

[34] K. Kohri, R. Narayan, T. Piran, Astrophys. J. 629, 341 (2005).

[35] M. Blasone, G. Vitiello, Ann. Phys. 244, 283 (1995).

[36] K. C. Hannabuss, D. C. Latimer, J. Phys. A 33, 1369 (2000).

[37] M. Blasone, A. Capolupo, S. Capozziello, S. Carloni, \& G. Vitiello, Phys. Lett A323, 182 (2004).

[38] Note that the corresponding temperature $T_{p} \sim 10^{-20} M_{\odot} / M_{p} \mathrm{GeV}$ [33]. 